\title{
Comparative study of neuroimaging features and clinical symptoms in patients with eclampsia
}

\author{
Neha Meena $^{1 *}$,Suman Meena ${ }^{1}$, Khushbu Meena ${ }^{1}$, Savitri Verma ${ }^{2}$
}

${ }^{1}$ Department of Obstetrics and Gynaecology, Government Medical College, Kota, Rajasthan, India
${ }^{2}$ Department of Obstetrics and Gynaecology, Jhalawar Medical College, Jhalawar, Rajasthan, India

Received: 03 August 2021

Accepted: 28 September 2021

\section{*Correspondence:}

Dr. Neha Meena,

E-mail: neha07.nm@gmail.com

Copyright: (c) the author(s), publisher and licensee Medip Academy. This is an open-access article distributed under the terms of the Creative Commons Attribution Non-Commercial License, which permits unrestricted non-commercial use, distribution, and reproduction in any medium, provided the original work is properly cited.

\begin{abstract}
Background: Eclampsia is a life-threatening emergency that remains a major cause for feto-maternal morbidity and mortality. The purpose of our study was to access various computed tomographic scan (CT) findings in eclampsia patients and compare neurological symptoms with radiological findings.

Methods: A prospective analytical study was undertaken in department of obstetrics and gynecology, J. K. Lon hospital, Kota during the period of 2019-2020. Women who presented as eclampsia and admitted in indoor wards were included in the study. Data analyzed included various maternal and fetal parameters, CT scan findings and outcome of pregnancy. Results: The incidence of eclampsia was $1.1 \%$ of total deliveries. High risk factors associated with eclampsia were primigravida (70\%), maternal age (70\% in 21-25 years age group), illiteracy (64\%), inadequate antenatal care (96\%), early gestation (68\%). On CT scan findings $52 \%$ patients had abnormal CT scan findings of which most common was cerebral edema (57.7\%). Parieto-occipital lobe was most common region to be affected. Altered sensorium was found to be significantly associated with abnormal CT scan findings.

Conclusions: Eclampsia is a major cause of fetal and maternal morbidity and mortality. CT scan in eclampsia have significant role in early diagnosis of patients with cerebral pathologies and these CT scan findings were associated with the level of consciousness and number of convulsive episodes. Thus, CT scan helps in further management of these patients by multidisciplinary approach.
\end{abstract}

Keywords: Eclampsia, CT scan, Blood pressure, Neurological complications in pregnancy

\section{INTRODUCTION}

Hypertensive disorders are one of the most common medical complications of pregnancy affecting between 5$10 \%$ of all gestations. ${ }^{1}$ It account for approximately a quarter of all antenatal admissions. These disorders continues to be the major cause of maternal and perinatal morbidity and mortality worldwide. ${ }^{2}$ Pre-eclampsia is estimated to affect about $3.9 \%$ of deliveries while eclampsia affects about $1.4 \%$ of deliveries. ${ }^{3}$ Due to improved medical care in the developed countries, rate of eclampsia is about 1 in 2,000 deliveries to 1 in 3448 pregnancies in the western world where as in developing countries the incidence of eclampsia is much higher between 1 in 30 to 1 in 500 deliveries. ${ }^{4}$ Mortality in eclampsia is around $1 \% .{ }^{1}$ In our institute, JK LON hospital Kota, incidence of gestational hypertension is $7.6 \%$ of total deliveries out of which $2.4 \%$ is preeclampsia and $1.1 \%$ is eclampsia.

Preeclampsia is a complex multi organ disorder characterized by pregnancy induced hypertension and proteinuria beyond 20 weeks (exception GTD and multiple pregnancy). Eclampsia is defined as new onset generalized tonic clonic seizures in pregnancy beyond 20-week gestation to 48 hours postpartum unrelated to other 
cerebral conditions. Eclampsia is preceded by preeclampsia in all cases but sometimes the signs of severe preeclampsia go unnoticed. ${ }^{1}$

Cerebral complications in eclampsia patients are major causes of death in these patients. Eclampsia along with hyper coagulopathy of pregnancy is a high-risk factor for patient in respect of development of cerebrovascular thrombosis/ ischemic strokes and intracranial haemorrhage..$^{5-9}$

The brain is normally protected from extremes of blood pressure by an auto regulation system of small arteries and arterioles that ensures constant perfusion over a wide range of systemic pressures, in response to the systemic hypotension, cerebral arterioles dilate to maintain adequate perfusion. Whereas vessels constrict in response to high pressure. Above the upper limit of auto regulations, hypertensive encephalopathy may occur.

The most common cause of convulsions developing in association with hypertension or proteinuria during pregnancy or immediately postpartum is eclampsia. Rarely, other aetiologies may mimic eclampsia it includes pre-existing epilepsy, cerebrovascular accidents (cerebral venous thrombosis, cerebral infarction, haemorrhage) space occupying conditions (tumour, brain abscess), CNS infections (meningitis, encephalitis), metabolic disorders (hypoglycaemia, hypocalcaemia, water intoxication), neurocysticercosis, amniotic fluid embolism, post-dural puncture cephalgia, and ruptured cerebral aneurysm during late pregnancy and the puerperium. A careful clinical evaluation and appropriate investigations would help to distinguish eclampsia from such conditions. Neuroimaging gives a more accurate assessment of the degrees of CNS involvement in these cases. ${ }^{10}$

The neurological symptoms seen in eclampsia are headache, visual abnormalities, vomiting, confusion, hyperreflexia, loss of consciousness, coma.

Various neurological changes occurring in eclampsia can be assessed by several neuro diagnostic tests such as electroencephalography (EEG), computed axial tomographic scan (CT), cerebral Doppler velocimetry, FLAIR, magnetic resonance imaging have been studied in women with eclampsia. On the basis of cerebral imaging findings, attention has been directed to hypertensive encephalopathy, cerebral oedema, infarction and cerebral haemorrhage as a model for the central nervous system abnormalities in eclampsia. ${ }^{11-13}$

CT scan is easily available, cost-effective imaging modality. The characteristic CT findings in eclampsia are: ${ }^{1}$ Normal, cerebral oedema, diffuse white matter low density areas, patchy area of low density, occipital white matter oedema, loss of normal cortical sulci, reduced ventricular size, acute hydrocephalus and cerebral haemorrhage: intraventricular haemorrhage and parenchymal haemorrhage (high density) and cerebral infarction: low attenuation areas and basal ganglia infarction.

In India eclampsia remains a high contributor towards maternal mortality and morbidity and also poor perinatal outcome. Thus, timely prediction of the onset of eclampsia and starting appropriate treatment as early as possible is important for favourable maternal and perinatal outcome. CT head is easily available in Indian scenario and very cost effective as compared to other neurological investigations. So far, a very few studies were done in Indian population on CT findings in eclampsia so this study was designed to know clinical significance of CT of head in eclampsia.

\section{METHODS}

This was a prospective, comparative and analytical study conducted at the tertiary centre, government medical college of Kota in one year from the period of 2019-2020. All eclampsia patients of any parity beyond 20 weeks of gestation up to 48 hours postpartum were taken as cases.

\section{Inclusion criteria}

All eclampsia patients (of gestation age beyond 20 weeks and blood pressure more than 140/90 mm of $\mathrm{Hg}$ ) were included in the study.

\section{Exclusion criteria}

Women who were known case of epilepsy, seizures due tometabolic disturbances, space occupying lesions, intracerebral infections, poisoning, trauma and women with gestation age $<20$ weeks were excluded from study.

Detailed geographical, obstetrical and medical history of patients including age, parity and gestational age, previous medical history and history of convulsion like duration, time, number of convulsion and presence of premonitory symptoms were sought.

General physical and obstetrical examination of patient was done. Routine investigations like blood pressure, urine for proteinuria measuring (by dipstick) and CBC, BT, CT, serum urea, serum creatinine, LFT, RFT were investigated. Standard $\mathrm{MgSO}_{4}$ protocol (Pritchard regime) given to all eclampsia patients. Termination of pregnancy was done either by induction of labour or caesarean section after assessing bishop score. CT scan of head was performed within 48 hours of confinement of foetus and after stabilizing the mother. Non-contrast-enhanced CT scan was performed with $5 \mathrm{~mm}$ and $10 \mathrm{~mm}$ section in the axial plain. The CT scan findings were collected, evaluated and compared to clinical sign and symptoms. Then combined neuroimaging and clinical diagnosis was assessed to see maternal outcome-whether mortality or morbidity. $\mathrm{P}<0.05$ is considered for statistical significance. Follow up CT scan was not performed as it was not included in the study protocol. 


\section{RESULTS}

A total of 50 patients with eclampsia were included in study who underwent CT scan of head. The most common age group to present as eclampsia was 21-25 years with $64 \%$ patients and the mean and standard deviation was 23.72 years and 3.28 years respectively.

The distribution of patients on the basis of sociodemographic profile demonstrates eclampsia was more common in patients belonging to rural areas $(68 \%)$, illiterates $(64 \%), 96 \%$ patients had un-booked pregnancy and $79.16 \%$ patients were referred (Table 1 ).

Table 1: Distribution of patients on basis of residence, education, antenatal care status, referral status.

\begin{tabular}{|llll|}
\hline \multirow{4}{*}{ Rariables } & & Frequency & $\%$ \\
& Urban & 16 & 32 \\
\cline { 2 - 4 } & Rural & 34 & 68 \\
\cline { 2 - 4 } & Total & 50 & 100 \\
\hline \multirow{3}{*}{ Education } & Literate & 18 & 36 \\
& Illiterate & 32 & 64 \\
\cline { 2 - 4 } & Total & 50 & 100 \\
\hline \multirow{2}{*}{$\begin{array}{l}\text { Pregnancy } \\
\text { status }\end{array}$} & Booked & 2 & 4 \\
\hline \multirow{2}{*}{$\begin{array}{l}\text { Referral } \\
\text { status }\end{array}$} & Un-booked & 48 & 96 \\
\cline { 2 - 4 } & Total & 50 & 100 \\
\hline & Referred & 38 & 79.16 \\
\cline { 2 - 4 } & Direct & 10 & 20.83 \\
\hline
\end{tabular}

Greater number of patients $(70 \%)$ were primigravida and only $30 \%$ patients were multigravida. Majority of patients $(56 \%)$ were from gestational age group of 29-36 weeks, $20 \%$ presented at term, $6 \%$ were less than 28 -week pregnancy and $18 \%$ were postpartum. $82 \%$ patients had antepartum eclampsia while $18 \%$ patients had postpartum eclampsia (Table 2).

Table 2: Distribution on the basis of parity and period of gestation.

\begin{tabular}{|lll|}
\hline Variables & Frequency & $\%$ \\
\hline Gravida & & \\
\hline Primi & 35 & 70 \\
\hline Multi & 15 & 30 \\
\hline Total & 50 & 100 \\
\hline POG (weeks) & & \\
\hline $20-28$ & 3 & 6 \\
\hline $29-36$ & 28 & 56 \\
\hline $37-40$ & 10 & 20 \\
\hline Post-partum & 9 & 18 \\
\hline Total & 50 & 100 \\
\hline
\end{tabular}

The $52 \%$ patients delivered through lower segment csection while $48 \%$ delivered vaginally. Out of these $68 \%$ delivered preterm while $32 \%$ delivered at term gestation. The $16 \%$ intrauterine deaths were observed (Table 3 ).
Table 3: Distribution on basis of mode of termination of pregnancy.

\begin{tabular}{|llll|}
\hline $\begin{array}{l}\text { Mode of termination } \\
\text { of pregnancy }\end{array}$ & Preterm & Term & Total \\
\hline Vaginal delivery & 14 & 10 & 24 \\
\hline \% & 28 & 20 & 48 \\
\hline LSCS & 20 & 6 & 26 \\
\hline \% & 40 & 12 & 52 \\
\hline Total & 34 & 16 & 50 \\
\hline
\end{tabular}

The $52 \%$ patients with eclampsia had abnormal changes detected on CT scan while $48 \%$ patients had normal CT scan findings. The most common $\mathrm{CT}$ scan finding observed in patients with eclampsia was $57.7 \%$ patients had cerebral oedema (PRES), 15\% had infarction, $11.53 \%$ showed sign of haemorrhage (Table 4). Majority of patients with $26.92 \%$ had parieto-occipital region affected. Parietal lobe was most commonly involved brain region $(53.85 \%)$ with maximum $26.92 \%$ sole parietal lobe involvement (Table 5).

Table 4: Frequency and distribution on the basis of CT scan findings.

\begin{tabular}{|lll|}
\hline CT scan findings & Frequency & $\%$ \\
\hline Cerebral edema & 15 & 57.7 \\
\hline Infarct & 4 & 15.4 \\
\hline Haemorrhage & 3 & 11.53 \\
\hline Cerebral ischemia & 1 & 3.84 \\
\hline ICH, PRES & 1 & 3.84 \\
\hline $\begin{array}{l}\text { B/L cerebral multiple } \\
\text { infarcts, PRES }\end{array}$ & 1 & 3.84 \\
\hline $\begin{array}{l}\text { Tentorial herniation, brain } \\
\text { stem twist }\end{array}$ & 1 & 3.84 \\
\hline Total & 26 & 100 \\
\hline
\end{tabular}

Table 5: Distribution of patients on the basis of lobar involvement on CT scan.

\begin{tabular}{|lll|}
\hline Area of brain involved & Frequency & $\%$ \\
\hline Parieto-occipital & 7 & 26.92 \\
\hline Temporo-occipital & 4 & 15.38 \\
\hline Parietal & 4 & 15.38 \\
\hline Temporal & 4 & 15.38 \\
\hline Parieto-temporal & 2 & 7.69 \\
\hline IVH & 2 & 7.69 \\
\hline Fronto-parieto-occipital & 1 & 3.86 \\
\hline Tentorial herniation & 1 & 3.86 \\
\hline Left ganglio capsular area & 1 & 3.86 \\
\hline
\end{tabular}

The $44 \%$ patients were in irritable/drowsy state at time of admission, rest were in conscious or unconscious state with $28 \%$ patients in each category. Maximum number patients $(64.28 \%)$ who had abnormal CT scan findings were those who were unconscious after convulsions at time of presentation. $\mathrm{P}=0.02$, which is statistically significant (Table 6). 
A significant association between gestational age and CT scan finding $(\mathrm{p}=0.008)$ was observed. Abnormal CT scan finding were more likely in patients with eclampsia in early pregnancy (Table 7).

There was significant association between systolic BP and MAP with CT scan finding with $\mathrm{p}=0.003$ and 0.025 respectively (Table 8 ).

The $52 \%$ patients had imminent signs before convulsions while $48 \%$ did not observed any symptom. Majority of patients with $57.14 \%$ had headache. The $7.14 \%$ had complaint blurring of vision, only $3.57 \%$ had epigastric pain. Out of 26 patients with imminent signs of eclampsia $46.2 \%$ patients had abnormal CT findings while $58.3 \%$ patients with no history of imminent signs had abnormal CT scan findings with $\mathrm{p}=0.389(>0.05)$, statistically significant association could not be proved (Table 9 ).

The $86 \%$ patients had less than 5 fits. $71.4 \%$ patients among those with $>5$ convulsions had abnormal CT scan findings. $(\mathrm{p}=0.267)$ (Table 10).

All patients of eclampsia were admitted in ICU/ HDU. Mechanical ventilation was required in $20 \%$ cases while $80 \%$ recovered spontaneously. The $52 \%$ cases required
ICU/ HDU stay for $<3$ days, the mean duration of stay was 3.58 days. Six percentage patients were shifted to neurology interventional ICU (NIICU) for further management while $6 \%$ mortality was noticed. Majority of patients who required mechanical ventilation had positive CT scan findings, $\mathrm{p}=0.007$, which is statistically significant. Also, most of patients who required ICU/HDU care $>5$ days had positive CT scan findings, $\mathrm{p}=0.04$, which is statistically significant (Table 11).

In our study $6 \%(3 / 50)$ mortality was recorded. First patient had antepartum eclampsia, was delivered by preterm cesarean section had associated antepartum hemorrhage, HELLP syndrome with acute renal failure and DIC. CT scan brain showed tentorial herniation with brain stem rotation and deviation of axis with cerebral edema. Second patient had antepartum eclampsia, unconscious at time of presentation, delivered by preterm cesarean section and later developed DIC, CT scan brain revealed acute parenchymal hemorrhage with intraventricular hemorrhage in all ventricles. Third patients presented with postpartum eclampsia and developed associated complications HELLP syndrome, acute renal failure and pulmonary edema (MODS) expired on 2 day of admission her CT scan showed intracranial hemorrhage with intraventricular hemorrhage on left side.

Table 6: Distribution on the basis of general condition of patient.

\begin{tabular}{|llllllll|} 
Conditions & $\mathbf{N}$ & $\%$ & CT normal & $\%$ & CT abnormal & $\%$ & P value \\
\hline Conscious & 14 & 28 & 11 & 78.57 & 3 & 21.42 & 0.02 \\
\hline $\begin{array}{l}\text { Irritable/ } \\
\text { Drowsy }\end{array}$ & 22 & 44 & 8 & 36.36 & 14 & 63.63 & 64.28 \\
\hline Unconscious & 14 & 28 & 5 & 35.71 & 9 & \\
\hline
\end{tabular}

Table 7: CT scan association with period of gestation.

\begin{tabular}{|llll|}
\hline CT & Mean (weeks) & SD & P value \\
\hline Normal & 35.667 & 2.389 & 0.008 \\
\hline Abnormal & 33.115 & 3.922 & \\
\hline
\end{tabular}

Table 8: CT scan association with blood pressure.

\begin{tabular}{|llllllll|}
\hline CT & Normal & \multicolumn{7}{c}{ Abnormal } & Mean & SD & P value \\
\hline SBP & N & Mean & SD & N & Men & 0.003 \\
\hline DBP & 24.00 & 153.33 & 17.11 & 26.00 & 169.62 & 16.37 & 0.243 \\
\hline MAP & 24.00 & 107.50 & 10.73 & 26.00 & 113.08 & 17.38 & 0.025 \\
\hline
\end{tabular}

Table 9: Distribution of patients on the basis of imminent signs of eclampsia.

\begin{tabular}{|c|c|c|c|c|c|c|}
\hline $\begin{array}{l}\text { Imminent } \\
\text { signs }\end{array}$ & $\mathbf{N}(\%)$ & Symptoms & Normal & Abnormal & Frequency (\%) & P value \\
\hline \multirow{4}{*}{ Present } & \multirow{4}{*}{$26(52)$} & Headache & \multirow{4}{*}{$14(53.8)$} & \multirow{4}{*}{$12(46.2)$} & 17 & \multirow{5}{*}{0.389} \\
\hline & & Vomiting & & & 9 & \\
\hline & & Blurring of vision & & & 2 & \\
\hline & & Epigastric & & & 1 & \\
\hline Absent & $24(48)$ & -- & $10(41.7)$ & $14(58.3)$ & - & \\
\hline
\end{tabular}


Table 10: Distribution of patients on the basis of number of convulsions and its association with CT scan findings.

\begin{tabular}{|c|c|c|c|c|c|}
\hline \multirow{2}{*}{\multicolumn{2}{|c|}{ Number of convulsions }} & \multicolumn{3}{|l|}{$\mathrm{CT}$} & \multirow{2}{*}{ P value } \\
\hline & & Normal & Abnormal & Total & \\
\hline \multirow{2}{*}{$<5$} & Count & 22 & 21 & 43 & \multirow{4}{*}{0.267} \\
\hline & $\%$ & 51.2 & 48.8 & 86 & \\
\hline \multirow{2}{*}{$>5$} & Count & 2 & 5 & 7 & \\
\hline & $\%$ & 28.6 & 71.4 & 14 & \\
\hline \multicolumn{2}{|c|}{ Total } & 24 & 26 & $50(100)$ & \\
\hline
\end{tabular}

Table 11: Distribution on the basis of critical care required and its association with CT scan, $(\mathrm{n}=50)$.

\begin{tabular}{|c|c|c|c|c|c|c|c|}
\hline Mechanical ventilation & Frequency & $\%$ & CT normal & $\%$ & CT abnormal & $\%$ & P value \\
\hline Present & 10 & 20 & 1 & 10 & 9 & 90 & \multirow{2}{*}{0.007} \\
\hline Absent & 40 & 80 & 23 & 57.5 & 17 & 42.5 & \\
\hline \multicolumn{8}{|l|}{ ICU duration (Days) } \\
\hline$<5$ & 43 & 86 & 23 & 53.48 & 20 & 46.51 & \multirow{2}{*}{0.04} \\
\hline$>5$ & 7 & 14 & - & 0 & 7 & 100 & \\
\hline Shifted to NIICU & 3 & 6 & - & & 3 & & \\
\hline Mortality & 3 & 6 & - & 0 & 3 & 100 & \multirow{3}{*}{0.007} \\
\hline Present & 10 & 20 & 1 & 10 & 9 & 90 & \\
\hline Absent & 40 & 80 & 23 & 57.5 & 17 & 42.5 & \\
\hline
\end{tabular}

\section{DISCUSSION}

In present study, 64\% (32/50) patients of eclampsia were from the age group of 21-25 years which is consistent with the results in other studies conducted by Pradhan et al, Rajeshwari et al and Agrawal et al. ${ }^{14-16}$

In present study, the maximum number of patients $(48 / 50)$ were un booked out of which $79.16 \%$ (38/48) were referred cases, $20.83 \%$ (10/48) came directly and only $4 \%$ patients were booked and had supervised pregnancy. Similar findings were observed in study by Jain et al, Parihar et al and Chibbar et al where majority of patients were un booked and lacked antenatal care. ${ }^{17-19}$ While Dasgupta et al in their study observed majority of eclamptic patients were booked pregnancy. ${ }^{20}$ In the present study the incidence of eclampsia was more among rural patients, 68\% (34/50) compared to urban area women, $32 \%$ (16/50). Our results were supported by study conducted by Agrawal et al, Jain et al and Haque et al. ${ }^{17,21,22}$ In the present study $64 \%$ (32/50) patients were illiterate and did not have primary education while $36 \%$ $(18 / 50)$ patients were literate. In contrast to this, the study conducted by Nazeen et al shows $17.7 \%$ patients were illiterate while $82.3 \%$ patients were literate. ${ }^{23}$ As majority of eclamptic patients were from rural area, due to their low socio-economic status and family background they did not had access to educational institute.

The patients from referred category did not had supervised pregnancy, thus timely diagnosis and management of gestational hypertension and preeclampsia was not done. While the booked patients could not recognize the signs of imminent eclampsia on time and became eclamptic.
The incidence of eclampsia was found to be more in primigravida than in multigravida in the present study, $70 \%(35 / 50)$ patients were primigravida. A similar outcome was shown in the studies conducted by Rajeshwari et al, Dasgupta et al, Brouh et al, Khandaker et al and Brewer et al. ${ }^{15,24-27}$ While Khandaker et al in their study found the incidence of eclampsia more in multigravida patients. ${ }^{25}$

In this study we observed $82 \%(41 / 50)$ patients were in antepartum state when became eclamptic while $18 \%$ (9/50) were postpartum. No patient had intrapartum seizures. When timing of eclampsia is considered, our study is in accordance with all the previous studies by Khandaker et al, Gurjar et al and Parihar et al this correlates with the fact that incidence of postpartum eclampsia is less than that of antepartum eclampsia, however the percentage of cases differ and this may be due to small number of sample size taken in studies. ${ }^{18,25,27}$

In the present study majority of patients with $56 \%(28 / 50)$ presented in the group where period of gestation was between 29-36 weeks. 20\% (10/50) cases presented at term pregnancy (37-40 weeks) while 6\% (3/50) cases had eclamptic convulsions in early preterm pregnancy in 20 28 weeks gestation. This was supported by the studies conducted by Khandaker et al, Rajeshwari et al and Pradhan et al where majority of patients had preterm pregnancy. ${ }^{14,15,25}$

In the present study $52 \%(26 / 50)$ patients delivered by cesarean section while $48 \%$ (24/50) delivered through normal vaginal route after induction of labor. Khandaker et al, Brewer et al, Chibbar et al and Dasgupta et al support same findings with majority of eclampsia patients 
delivered by cesarean section. ${ }^{19,20,25,26}$ While Parihar et al shows greater number of normal deliveries comparatively. ${ }^{18}$ This is because maximum number of patients had eclampsia in 29- 36 weeks of gestation with poor bishops score so delivery of these patient with induction of labor would have taken more time which might have resulted in further deterioration of patient condition. Besides that, consequences of lack of antenatal care are further compounded and as a result of which these patients receive the obstetric care in emergency often resulting in surgical delivery. This formed over half of the patients in this study which were similar to other reports.

In present study, normal CT scan of head was found in $48 \%(24 / 50)$ cases while abnormal findings were present in $52 \%(26 / 50)$ patients of which $57.7 \%(15 / 26)$ had cerebral edema, $15.4 \%$ (4/26) had infarct, $11.53 \%(3 / 26)$ had hemorrhage and cerebral ischemia, intracranial hemorrhage with PRES and bilateral cerebral multiple infarcts with PRES were present in $3.84 \%$ (1/50) patients each. This observation is supported by studies conducted by Khandekar et al, Harandou et al, Akan et al and Dasgupta et al showing majority of patients having abnormal findings on CT scan of head with cerebral edema as most common finding. ${ }^{20,25,26,28}$ Pradhan et al in their study observed equal number of patients had normal and abnormal CT scan findings. ${ }^{14}$ While Millez et al in their study observed $41 \%$ patients to have abnormal CT scan findings. ${ }^{30}$

Parietal lobe was the most common brain region involved in present study. The $26.92 \%(7 / 26)$ patients had parietooccipital lobe involvement while temporo-occipital, parietal and temporal lobe involvement seen in $15.38 \%$ (4/26) patients in each. The $3.86 \%(1 / 26)$ patients had fronto-parieto-occipital lobes involvement, tentorial herniation, and left ganglio-capsular region. Similar results were observed by Pradhan et al, Rajeshwari et al, Khandaker et al, Gurjar et al while Dasgupta et al in their study found diffuse brain involvement to be the most common finding. ${ }^{14,15,20,25,27}$

In present study mean SBP was $153.33 \mathrm{~mm}$ of $\mathrm{Hg}$ in CT normal patient group and $169.62 \mathrm{~mm}$ of $\mathrm{Hg}$ in CT abnormal patient group $(\mathrm{p}=0.003)$ with statistically significant association. Mean diastolic BP in CT normal group was $107 \mathrm{~mm}$ of $\mathrm{Hg}$ and in CT abnormal group was $113.08 \mathrm{~mm}$ of $\mathrm{Hg}(\mathrm{p}=0.243)$. MAP in CT normal group was $122.58 \mathrm{~mm}$ of $\mathrm{Hg}$ and in $\mathrm{CT}$ abnormal group was $132.80 \mathrm{~mm}$ of $\mathrm{Hg}$ with $\mathrm{p}=0.025$, which is statistically significant.

Rajeshwari et al in they observed no significant association between CT scan finding and blood pressure. $(p>0.005) .{ }^{15}$

In present study no association was found between area of lesion and imminent symptom. While Khandaker et al in their study observed significant association between some of neurological symptoms (coma and visual disturbances) and CT scan finding. ${ }^{25}$ This may be due to the fact most of the patients presented in irritable/ unconscious state and were not able to recall their symptoms properly.

In present study $46.2 \%(12 / 26)$ patients who had imminent signs of eclampsia had abnormal CT scan findings while $58.3 \%$ (14/24) with no signs of imminent eclampsia before seizures had CT abnormal findings showing no association in preceding symptoms with CT scan ( $\mathrm{p}=0.389)$.

While Pradhan et al in their study observed significant difference between CT scan findings of patients with neurological symptoms from those who have no neurological symptoms. ${ }^{14}$

Twenty percentage (10/50) patients in present study required intubation/mechanical ventilation while $80 \%$ (40/50) patients recovered spontaneously without mechanical ventilation. The $86 \%(43 / 50)$ patients required ICU/HDU stay for 5 or $<5$ days while $14 \%$ (7/50) patients stayed for more 5 days, $6 \%$ (3/50) patients were shifted to neurology ICU for further management.

Parihar et al observed in their study that $41 \%$ patients received mechanical ventilation while $90 \%$ patients received oxygen and advance monitoring, the average

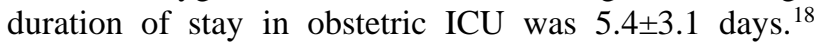
$17.93 \%$ mortality rate was noted. While in study by Brouh et al the time of care spent in the ICU was $07.13 \pm 4.18$ hours (range, $03-20$ hours). ${ }^{24}$ The $46.15 \%$ patients needed mechanical ventilation while in $76.9 \%$ patient tracheal intubation was performed.

\section{CONCLUSION}

Eclampsia and preeclampsia remain a major cause of maternal and perinatal morbidity and mortality in developing world. Inadequate antenatal care, delay in women seeking help, in diagnosis and in referral from peripheral referral centers are major cause for poor outcome. Iatrogenic prematurity is the major cause of prematurity leading to perinatal mortality in eclamptic patients. Timely diagnosis depending upon imminent signs and symptoms and early intervention in high-risk cases can improve maternal outcome in eclampsia. CT scan in eclampsia have a significant role in early diagnosis of patients with cerebral lesions and CT findings are associated with the level of consciousness and number of convulsive episodes. Thus, CT scan helps in further management of these patients by multidisciplinary approach. So, it is emphasized to include CT scan in investigation protocol for eclampsia management in patients with altered sensorium.

\section{Funding: No funding sources Conflict of interest: None declared \\ Ethical approval: The study was approved by the Institutional Ethics Committee}




\section{REFERENCES}

1. Hypertensive disorders in pregnancy. Williams Obstetrics, $24^{\text {th }}$ edition. McGraw-Hill Companies Inc. 2014;40;731.

2. Das R, Biswas S. Eclapmsia: the major cause of maternal mortality in Eastern India. Ethiop $\mathbf{J}$ Health Sci. 2015;25(2):111-6.

3. Abalos E, Cuesta C, Grosso AL, Chou D, Say L. Global and regional estimates of preeclampsia and eclampsia: a systematic review. Eur J Obstetr, Gynecol, Reproduct Biol. 2013;170(1):1-7.

4. Matter F, Sibai BM. Eclampsia viii risk factors for maternal morbidity. Am J obst gynecol. 2000;182:30712.

5. Leitch CR, Cameron AD, Walker JJ. The changing patterns of eclampsia over a 60 -year period. Br J Obstet Gynaecol. 1997;104: 917-22.

6. Mass JL, Lamy C. Stroke in pregnancy in postpartum period. In: Ginsberg MD, Bogousslavsky J, editors. Cerebrovascular disease: pathophysiology, diagnosis and management. Malden: Blackwell sci. 1988;1684-97

7. Kanki T, Mihara F, Nakanoh. Diffusion weighted images and vasogenic edema in pregnancy. Obstet Gynaecol. 1999;93:821-3.

8. Zeeman G. Flankenstein JJ, Twickler DM. Cerebral infarction in eclampsia. Am J Obstet Gynaecol. 2002;100:140.

9. Manfredi M, Baltramello A. Eclamptic encephalopathy, neuroimaging and pathological consideration. Acta neurol Scand. 1997;96:277-82.

10. Gabbe Sibai BM. Hypertension, $6^{\text {th }}$ edi. Section 6 . 2009;35:852.

11. Marques R, Braga J, Leite I, Jorge CS. Neurological involvement in preeclampsia/ eclampsia: the role of neuroimaging. Acta Med Port. 1997;10:585-8.

12. Harandou M, Madani N, Labibe S, Messouak O, Boujraf $\mathrm{S}$, Benkirane $\mathrm{S}$ et al. [Neuroimaging findings in eclamptic patients still symptomatic after 24 hours: a descriptive study about 19 cases. Ann Fr Anesth Reanim. 2006;25:577-83.

13. Akan H, Küçük M, Bolat O, Selçuk MB, Tunali G. The diagnostic value of cranial computed tomography in complicated eclampsia. J Belge Radiol. 1993;76:304-06.

14. Pradhan K, Nath SS, Panigrahi SK. Intracerebral changes detected by CT scan of brain in post-delivery eclampsia patients. EJPMR. 2018;5(11):468-75.

15. Rajeswari KSR. Computed tomography scan findings in eclampsia. Int J Reprod Contracept Obstet Gynecol. 2018;7(11):4432-8.

16. Agarwal M, Gautam A. study of fetomaternal outcome in eclampsia. Int J Reprod Contracept Obstet Gynecol. 2020;9(10):4155-9.

17. Jain R, Bindal J. Maternal and perinatal outcomes in eclampsia: a retrospective analysis in a referral hospital.
Int J Reprod Contracept Obstet Gynecol. 2017;6(7):2806-11.

18. Parihar BC, Yadav B, Patel J. Critical care management of eclampsia patients - one year study. Int J Reprod Contracept Obstet Gynecol. 2020;9(12):4850-4.

19. Chibber R, Al-Hijji J, Amen A, Fouda M, Kaleemullah ZM, El-Saleh E, Mohammed AT. Maternal and perinatal outcome of eclampsia over a decade at a tertiary hospital in Kuwait. J Maternal-Fetal Neonatal Med. 2016;29(19):3132-7.

20. Dasgupta A, Biswas SK, Jana SK, Nandy D. Brain CT scans findings in Eclampsia mothers and its clinical evaluation: a cross sectional study. JMSCR. 2019;7:4.

21. Agrawal S, Walia GK, Staines-Urias E, Casas JP, Millett C. Prevalence of and risk factors for eclampsia in pregnant women in India. Family Med Community Health. 2017;5(4):225-44.

22. Haque H, Thapa K. Maternal and Fetal Outcome in Eclampsia: A Study from Tertiary Care Hospital. JNGMC. 2017;15:2-17.

23. Nazneen N, Laskar MS, Barua PP, Haque SD. Factors influencing pregnancy induced hypertension in Khulna Medical College Hospital. Mediscope. 2016;3(2):31-5.

24. Brouh Y, Jean KK, Ouattara A, Tetchi Y, Pete Y, Koffi $\mathrm{N}$ et al. Brain lesions in eclampsia. Indian $\mathrm{J}$ Crit Care Med. 2016:20(3):178-81.

25. Khandaker S, Haldar M, Munshi S. Intra Cerebral Changes Detected by CT scan of Brain in Eclampsia. Austin J Obstet Gynecol. 2014;1(3):4.

26. Brewer J, Owens MY, Wallace K, Reeves AA, Morris R, Khan $M$ et al. Posterior reversible encephalopathy syndrome in 46 of 47 patients with eclampsia. Am J Obstet Gynecol. 2013;208(6):468.e1-6.

27. Gurjar B, RP Rawat. CT scan findings in patients of eclampsia. Int J Reprod Contracept Obstet Gynecol. 2017;6(8):3405-08.

28. Harandou M, Madani N, Labibe S, Messouak O, Boujraf $\mathrm{S}$, Benkirane $\mathrm{S}$ et al. Neuroimaging findings in eclamptic patients still symptomatic after 24 hours: a descriptive study about 19 cases. Ann Fr Anesth Reanim. 2006;25:577-83.

29. Akan H, Küçük M, Bolat O, Selçuk MB, Tunali G. The diagnostic value of cranial computed tomography in complicated eclampsia. J Belge Radiol. 1993;76:304-06.

30. Milliez J, Dahouna, Boudraa M. Computed tomography of the brain in eclampsia. Obstet Gynecol. 1990;75:97580.

Cite this article as: Meena N, Meena S, Meena K, Verma S. Comparative study of neuroimaging features and clinical symptoms in patients with eclampsia. Int J Reprod Contracept Obstet Gynecol 2021;10:4171-7. 